Artículo Original

\title{
Índices de disnea de Borg y MRC percibida en pacientes COVID-19 persistente con hipertensión pulmonar
}

\section{Indices of Borg dyspnea and perceived CRM in persistent COVID-19 patients with pulmonary hypertension}

https://doi.org/10.52808/bmsa.7e5.61e2.008

Raul Germán Castro Garcia ${ }^{1,2, *}$ https://orcid.org/0000-0003-4315-2031

Lizandro Nicolas Anzules Cuzco ${ }^{3,4}$

https://orcid.org/0000-0003-3887-884X

Andrea Judith Rubira Clavijo5

https://orcid.org/0000-0002-0540-232X

Erick John Sánchez Acuña ${ }^{3.4}$

https://orcid.org/0000-0003-2530-3879

José Raul Morocho Anchatuña ${ }^{6,7}$

https://orcid.org/0000-0001-9470-9384

Jimmy Eduardo Carreño Ramos ${ }^{8}$

https://orcid.org/0000-0002-1591-1005

José Domingo Valle Pinargote ${ }^{8}$

https://orcid.org/0000-0002-7464-9000

Mailin Daniela Barberan Quiroz ${ }^{7}$

https://orcid.org/0000-0003-4888-555X

Montesdeoca Pazmiño María Lisbeth ${ }^{7}$

https://orcid.org/0000-0001-9843-674X

Raúl Augusto Peñaherrera Cepeda ${ }^{7}$ https://orcid.org/0000-0001-9342-5697

Carlos Julio Moncayo Valencia ${ }^{9}$ https://orcid.org/0000-0002-0548-1638

Ana Gabriela Niola Toasa ${ }^{7}$

Recibido: 30/04/2021

https://orcid.org/0000-0002-4033-9522

Aceptado: 19/07/202

\section{RESUMEN}

El SARS-CoV-2 causante de la enfermedad COVID-19, clínicamente se oscila desde episodios leves seudogripales a otros graves e incluso potencialmente mortales, sobre todo, debido a la insuficiencia respiratoria aguda, independientemente de la gravedad se puede superar el curso agudo, sin embargo, la persistencia de síntomas o complejo sintomático multiorgánico, denominado COVID persistente. Dentro de estos síntomas persistente esta la dificultad respiratoria. Se realizó un estudio descriptivo, observacional y transversal en 29 personas diagnosticadas de COVID-19 persistente más comorbilidad hipertensión pulmonar, entre 2019 y 2021. Se aplicaron las escalas de disnea de Borg en reposo y por prueba de esfuerzo (Prueba de la caminata en 6; y la escala de disnea del Medical Research Council. IFB B categorizan entre 0 y 2 y R2 alto $(0,7993)$ explica la percepción de malestar respiratorio que aparece con niveles de actividad que normalmente no causan este tipo de malestar (actividad diaria), y puede ponderarse por el nivel de actividad necesario para que se desencadene. Ambas escalas tienen validez, fiabilidad, especificidad, sensibilidad y valor predictivo para el uso que se ha establecido, de fácil uso y compresión.

Palabras clave: IBF, MHC, disnea, COVID persistente.

\section{ABSTRACT}

The SARS-CoV-2 that causes the COVID-19 disease, clinically ranges from mild flu-like episodes to other serious and even life-threatening, especially due to acute respiratory failure, regardless of the severity the acute course can be overcome, however, the persistence of symptoms or multi-organ symptom complex, called persistent COVID. Among these persistent symptoms is respiratory distress. A descriptive, observational and cross-sectional study was carried out in 29 people diagnosed with persistent COVID-19 plus comorbidity pulmonary hypertension, between 2019 and 2021. The Borg dyspnea scales were applied at rest and by stress test (Walk test in 6 ; and the Medical Research Council dyspnea scale. IFB B categorizes between 0 and 2 and high R2 (0.7993) explains the perception of respiratory discomfort that appears with activity levels that do not normally cause this type of discomfort (daily activity), and can be weighted by the level of activity necessary for it to be triggered.Both scales have validity, reliability, specificity, sensitivity and predictive value for the use that has been established, easy to use and understand.

Key words: IBF, MHC, dyspnea, persistent COVID. 


\section{Introducción}

El SARS-CoV-2 causante de la neumonía viral grave denominada enfermedad COVID-19, clínicamente se oscila desde episodios leves seudogripales a otros graves e incluso potencialmente mortales, sobre todo, debido a la insuficiencia respiratoria aguda, como lo señala Castro et al., (2021) y Diaz et al., (2021). Ahora bien, independientemente de la gravedad un sin número de personas que han superado el episodio agudo de la COVID-19, sin embargo, la persistencia de síntomas que pueden ser prolomgados incluso por meses, nos comlleva a caracterizar el nuevo "síndrome" o complejo sintomático multiorgánico, denominado COVID persistente o Long COVID (Carfì et al., 2020; Simonelli et al., 2021). Para Sociedad Española de Médicos Generales y de Familia (SEMG), (2000) y Sanz Almazán et al., (2021) los síntomas y signos más frecuentemente descritos son astenia, malestar general, cefalea, bajo estado de ánimo, dolores articulares, falta de concentración, entre otros, hasta alcanzar una lista con más de 200 síntomas diferentes.

Además de las consecuencias fisiológicas, se reportan una alta prevalencia de deterioro en el rendimiento físico en pacientes que se recuperan de COVID-19 (Arnanz González et al., 2021). Así este grupo de autores, reportan en pacientes sin discapacidades previas, se encontró que la contracción voluntaria máxima para cuádriceps y bíceps era $54 \%$ y $69 \%$ de los valores predichos, respectivamente y el $76 \%$ de los pacientes informaron al menos un síntoma y el $23 \%$ informaron ansiedad o depresión hasta 6 meses después de la infección aguda. Los síntomas más comunes fueron fatiga, debilidad muscular o dificultades para dormir. Al respecto Sanz Almazán, (2021) señala que más del $10 \%$ de los pacientes que han sufrido COVID-19 presentan síntomas persistentes a lo largo del tiempo, que limitan en gran medida su calidad de vida y suponen consecuencias muy desfavorables para los sistemas de salud.

En otro orden de ideas, la disnea se define como una experiencia subjetiva de falta de confort al respirar (dificultad en la respiración); una percepción de mayor trabajo respiratorio, que aparece, tanto en reposo como con un grado de actividad física inferior al esperado; engloba sensaciones cualitativamente diferentes y de intensidad variable (Dyspnea 1999; Rose, 1999 y Mularski et al., 2016). Esta funcion vital es regulada tanto por factores voluntarios como dependientes del tronco encefálico, medida por los receptores periféricos alcanza la consciencia, el arco reflejo va desde los receptores sensoriales hasta los centros respiratorios automáticos de la médula, y de allí la percepción cerebral. Todo este mecanismo esta influido por ñps receptores en la vía aérea, el parénquima pulmonar, músculos respiratorios y quimiorreceptores proporcionan un feed-back sensorial a la médula espinal y centros nerviosos superiores por medio de una inervación vagal, frénica e intercostal.

Carfî et al. (2020) y Simonelli et al., (2021) en pacientes COVID-19 al referirse a la disnea, indican que se produce cuando una persona siente dificultad o incomodidad a la hora de respirar, así como la sensación de no estar recibiendo suficiente aire, el corazón es incapaz de bombear suficiente sangre para suministrar oxígeno al cuerpo. Sin embargo, es muy subjetivo, ya que no todas las personas respiran de la misma forma, pero el individuo puede identificarla al sentir ahogo o falta de aire mientras realiza un determinado ejercicio o actividad que antes toleraba bien. Aunado a ello estan las comorbobilides, entre ellas la hipertensión pulmonar, ésta es el aumento de presión en la circulación pulmonar, puede ser secundaria a muchas causas; algunos casos son idiopáticos, los vasos sanguíneos pulmonares se contraen y/o se obstruyen, y conduce a la sobrecarga grave ventricular derecha y a la insuficiencia (Gladwin \& Levine, 2020). Comunmente se presenta astenia, disnea con el ejercicio y, en ocasiones, malestar torácico y síncope.

Habitualmente los pacientes con hipertensión arterial pulmonar (HAP) cuando son hospitalizados, suelen tener peores evoluciones si se comparan con otras patologías. Por ello, la actual pandemia por COVID-19 ha supuesto un desafío específico para estos pacientes (Ryan et al., 2020). En pacientes COVID-19, Avellanas Chavala, (2020) señala que la vasoconstricción arterial pulmonar acompañada, en dependencia de la etiología, de una hipoxemia de tolerancia variable, que puede llegar a ser severa; la disfunción endotelial y el remodelado vascular son dos procesos importantes presentes en el desarrollo de la HAP. Cabría pensar que las alteraciones del endotelio con sus consecuencias trombóticas, que se están viendo en la fase hiperinflamatoria de la COVID-19, no sean tan floridas en los pacientes con HAP al estar previamente presente una disfunción endotelial, sin olvidar que estamos hablando de un estado de hipoxia crónica y, por tanto, hay que recordar los efectos fisiológicos, entre otros citoprotectores y de antiagregación plaquetaria. 
Los pacientes COVID persostente presentan síntomas fluctuantes con repercusión física, laboral y emocional. De allí que, es importante para la valoración este complejo proceso sintomático disponer de instrumentos de medida (escalas) que nos permitan poder cuantificar estos síntomas y sus fluctuaciones. Para ello, el grupo de trabajo de COVID persistente de la SEMG, (2021) tras revisar la bibliografía existente hasta el momento, decidió agrupar la sintomatología de la COVID persistente susceptible de uso de escalas en 8 grupos: calidad de vida, fatiga, dolor, trastornos emocionales, ejercicio físico, disnea, trastornos del sueño y alteraciones cognitivas.

Las escalas de medición en salud son herramientas que permiten evaluar características físicas, psicológicas o sociales que en ocasiones no pueden medirse de forma objetiva, y sirven de apoyo en la práctica clínica de los profesionales sanitarios (Martín Lesende et al., 2016). Aunque muy útiles en la toma de decisiones, en ningún caso sustituyen a la valoración clínica, para la selección debe considerarse diversos aspectos: 1) validez, fiabilidad, especificidad, sensibilidad y valor predictivo para el uso que se ha establecido; 2) Seleccionarla correcta para objetivo; 3) sencilla y breve; 3) facilidad de uso; y 4) adaptada al nivel cultural del paciente. SEMG, (2021) señalan que hay múltiples escalas disponibles, pero se priorizó que se ajustaran a las características de los pacientes con COVID persistente, con una visión global de los síntomas y que fueran sencillas y breves, de manera que se pudieran aplicar en la consulta diaria a pesar de la elevada carga asistencial.

Por lo tanto, la necesidad de medidas validadas es de suma importancia, utilizando equipos y procedimientos seguros, para evaluar las consecuencias a corto y largo plazo del COVID-19 (Sanz Almazán et al., 2021). Igualmente indican que las Escalas de valoración en pacientes con COVID persistente, pueden ser para calidad de vida, dolor, disnea, fatiga, ejercicio físico, trastornos del sueño, cognotivas y trastornos emocionales. En referencia ala disnea recomiendan las Escala Modified Medical Research Council (mMRC), Escala de la New York Heart Association NYHA y Escala de Borg. Revisamos las medidas utilizadas para evaluar el rendimiento físico en estos pacientes, los objetivos de nuestra investigación fueron las medidas de los síntomas inducidos por el ejercicio o las actividades de la vida diaria en los Índices de disnea de Borg y MRC percibida en pacientes COVID-19 persistente con hipertensión pulmonar.

\section{Materiales y Métodos}

Se realizó un estudio descriptivo, observacional y transversal en 29 personas diagnosticadas de COVID-19 persistente más comorbilidad hipertensión pulmonar, del hospital Teodoro Maldonado de la Ciudad de Guayaquil, desde marzo 2020 a abril, 2021. La totalidad de estos pacientes presentaron un cuadro clínico prolongado de debilidad muscular, astenia y disnea, semejante a la sintomatología descrita en los cuadros de fatiga crónica.

\section{Índice de fatiga de Borg antes del ejercicio o basal (IFB B) y después del ejercicio o final (IFB F)}

Se aplicaron las escalas de disnea de Borg (Borg, 1973), en reposo (Percepción de disnea durante las actividades de la vida diaria) y por prueba de esfuerzo (Prueba de la caminata en 6 minutos o PCT6); y la escala de disnea del Medical Research Council (MRC). La Escala Modificada de Borg de esfuerzo percibido calcula el grado de esfuerzo que un individuo percibe al hacer ejercicio. Esta tiene una progresión de 0 a 10, en la cual el valor mínimo se expresa con 0 el valor nulo de disnea y el 10 el valor casi máximo (disnea muy severa), puede categorizar un valor intermedio "0,5" cuando es casi imperceptible el cuadro disneico. Se utiliza únicamente durante la prueba de caminata ya que evalúa disnea aguda o relacionada con una actividad específica (Tabla 1).

Tabla 1. Escala de Borg

\begin{tabular}{|c|c|c|c|c|c|c|c|c|c|c|}
\hline \multicolumn{10}{|c|}{ Escala de Borg Modificada } \\
\hline $\mathbf{0}$ & $\mathbf{1}$ & $\mathbf{2}$ & $\mathbf{3}$ & $\mathbf{4}$ & $\mathbf{5}$ & $\mathbf{6}$ & $\mathbf{7}$ & $\mathbf{8}$ & $\mathbf{9}$ & $\mathbf{1 0}$ \\
\hline Reposo & $\begin{array}{c}\text { Muy muy } \\
\text { suave }\end{array}$ & $\begin{array}{c}\text { Muy } \\
\text { Suave }\end{array}$ & Suave & $\begin{array}{c}\text { Algo } \\
\text { duro }\end{array}$ & Duro & Más duro & $\begin{array}{c}\text { Muy } \\
\text { duro }\end{array}$ & $\begin{array}{c}\text { Muy } \\
\text { muy duro }\end{array}$ & $\begin{array}{c}\text { Máximo } \\
\text { Extremadamente } \\
\text { máximo }\end{array}$ \\
\hline
\end{tabular}

Fuente: Borg, (1973). Modificada

Los resultados del IFB F se categorizaron en una escala de ordinal por intervalos de clases (Tabla 2), agrupándose en 4 clases, según los criterios técnicos sugeridos por Martínez Hernández et al., (2016):

Tabla 2. Escala de categorización de Índice de fatiga de Borg antes del ejercicio o basal

\begin{tabular}{|c|c|c|c|c|c|c|c|c|c|c|}
\hline \multicolumn{1}{|c|}{ Escala de categorización de IFB F } \\
\hline Nada & \multicolumn{1}{|c|}{ Leve } & Moderada & \multicolumn{7}{|c|}{ Severa } \\
\hline $\mathbf{0}$ & $\mathbf{1}$ & $\mathbf{2}$ & $\mathbf{3}$ & $\mathbf{4}$ & $\mathbf{5}$ & $\mathbf{6}$ & $\mathbf{7}$ & $\mathbf{8}$ & $\mathbf{9}$ & $\mathbf{1 0}$ \\
\hline
\end{tabular}

\section{Cuantificación de disnea - Escala de disnea modificada del Medical Research Council (mMRC)}

La escala de disnea del Medical Research Council (mMRC) o escala de Fletcher de 1952 revisada por Schilling en 1955 (Fletcher, 1959). La categorización de esta escala es de 0 a 4. Siendo 0: La disnea se produce solo 
con un gran esfuerzo físico; 1: La disnea se produce al andar deprisa en llano o al subir una pendiente poco pronunciada; 2: La disnea imposibilita mantener el paso de otras personas de la misma edad caminando en llano, $\mathrm{u}$ obliga a detenerse o descansar al andar en llano al propio paso; 3: Tiene que detenerse a descansar al andar $100 \mathrm{~m}$ o a los pocos minutos de andar en llano y 4: La disnea impide al paciente salir de casa o aparece con actividades tales como vestirse o desvestirse.

\section{Saturación de oxígeno (SpO2)}

En el estudio se determinó el porcentaje de saturación de oxígeno de la sangre arterial medido con un oxímetro de pulso modelo OXI- 9800. La misma se clasificó en:

1. Saturación de oxígeno conservada: después de practicarse la PCT6 no sufre variaciones o disminuye menos de un $4 \%$

2. Desaturación: cuando el por ciento de $\mathrm{SpO}_{2}$ después de practicarse la PCT6 desciende un $4 \%$ o más en relación a la $\mathrm{SpO}_{2}$ medida antes de realizada la PCT6.

\section{Distancia Recorrida (DR)}

La distancia en metros recorrida por el paciente en la PCT6 como parámetro que orienta la gravedad de la dificultad respiratoria, se registró categorizándose en puntajes des " 0 " a "4", donde " 0 " corresponde a una distancia de recorrido mayor a 350 metros. " 1 " entre 250 a 349; " 2 " entre 150 a 2449; y “3” menor a 150 metros.

Se utilizó estadística descriptiva con medidas de tendencia central para el análisis de los datos sobre el comportamiento del IFB, antes y después de la realización de la prueba de marcha de los 6 min (PCT6) y su relación con la saturación de oxígeno al final de la prueba y con la distancia recorrida en la misma.

\section{Resultados}

La totalidad de los pacientes COVID-19 persistentes $(\mathrm{N}=29)$ en los estudios radiológicos de tórax, mostraron agrandamiento del ventrículo (hipertrofia y dilatación) y aurícula derecha (dilatación) del corazón y/o de las arterias pulmonares, características de la hipertensión pulmonar. Por otra parte, en los electrocardiogramas se revelaron signos de agrandamiento o distensión del ventrículo derecho, manifestarse con ondas $\mathrm{P}$ de mayor amplitud (P pulmonar) y depresión del segmento ST en las derivaciones II, III y aVF, desviación hacia la derecha del eje QRS, desviación inferior del vector de la onda $\mathrm{P}$ y disminución de la progresión de las ondas $\mathrm{R}$ en las derivaciones precordiales.

En los Eco Doppler Color, en el 100,00\% de los pacientes se evidencio regurgitación tricuspídea insuficiencia pulmonar. presión sistólica de arteria pulmonar elevada, (tiempo de aceleración máxima < 100 msgds (milisegundos), E/A (relación onda E / onda A) 1 en flujo mitral.

\section{IFB B e IFB F en pacientes COVID-19 con hipertensión pulmonar}

El sexo femenino represento en este estudio el 79,31\% (23/209) de la muestra total. En referencia al sexo los promedios fueron de 46,55 y 37,31 años para el sexo femenino y masculino respectivamente; cuyos rangos de edad oscilan entre 14 y 70 años (Tabal 3). El Índice de Masa Corporal (IMC) arrojo valores promedios de 25,31 en mujeres, lo que significa leve exceso de peso corporal, debido que 11 femeninas evidenciaron sobrepeso de moderado a severo.

El IFB B promedio fue de 0,89 y 1,26 para mujeres y hombres respectivamente, es decir, los síntomas de dificultad respiratoria de categorizan entre 0 y 2 (nulos a muy suaves) como se muestra en la figura 1., estos se intensifican tras la actividad diaria arrojando categorizaciones de moderado a severo en el 69,97\% (20/29) de los casos. El $\mathrm{R}^{2}$ o coeficiente de determinación alto $(0,7993)$ nos explica la respuesta de variable disnea, como predictor al intensificar la actividad de rutina como vestirse y desvertirse, o camina en plano o en leves inclinaciones.

Tabla 3. IFB B e IFB F con actividad diaria en pacientes COVID-19 con hipertensión pulmonar

\begin{tabular}{lccccccccc}
\hline & \multicolumn{3}{c}{ Femenino } & N=23 & \multicolumn{4}{c}{ Masculino N=6 } \\
\cline { 3 - 10 } & & Media & Des Est & Max & Min & Media & Des Est & Max & Min \\
\hline \multirow{2}{*}{ Edad } & 46,55 & 13,69 & 70 & 19 & 37,31 & 26,12 & 70 & 14 \\
IMC & 25,31 & 5,54 & 39 & 17 & 21,54 & 13,91 & 39 & 6 \\
\multirow{2}{*}{ Escala de Borg } & Reposo (IFB B) & 0,89 & 1,13 & 3 & 0 & 1,26 & 1,26 & 3 & 0 \\
& Act, diaria (IFB F) & 4,19 & 2,94 & 10 & 0 & 4,28 & 4,20 & 10 & 0 \\
\hline
\end{tabular}

IFB B: Índice de fatiga de Borg antes del ejercicio o basal.

IFB F: Índice de fatiga de Borg después del ejercicio o final. 


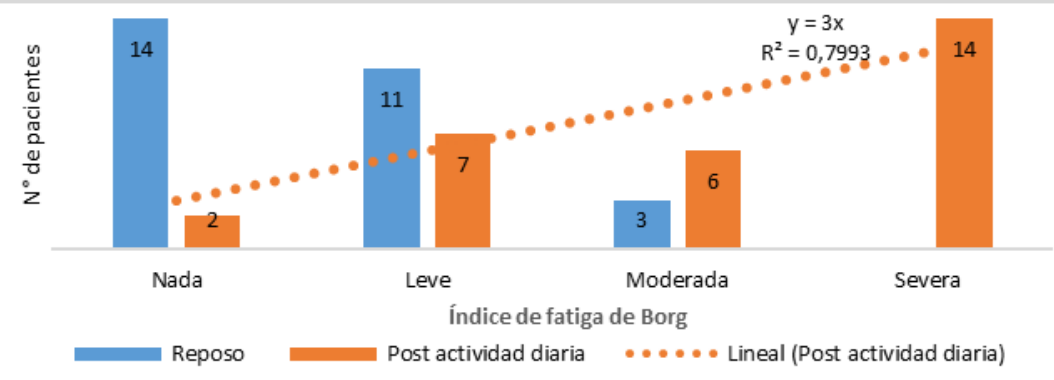

Figura 1. Categorización IFB B e IFB F con actividad diaria en pacientes COVID-19 con hipertensión pulmonar

Los IFB B e IFB F con prueba de la caminata se muestran en la tabla 4., en mujeres y hombres en reposo se obtuvieron valores promedios de IFB B de 1,14 y 1,95 respectivamente, siendo categorizadas como ausencia hasta muy leve dificultad respiratoria, sin embargo, 8 de 29 pacientes desistieron de la prueba por capacidad respiratoria reducida (Fatiga severa). Posterior a la PCT6 el IFB F arrojo promedios de 3,38 y 3,53 para mujeres y hombres respectivamente, categorizándose de moderado a severo en 14,57\% (12/21) de los casos, que presentaron sensación de fatiga después del ejercicio. Por su parte el $\mathrm{R}^{2}$ nos indica la relación de intensidad de esfuerzo versus demanda respiratoria, observando en la figura 2., el desplazamiento de la muestra a categorías más severas.

La respuesta percibida por la IFB B e IFB F, se ratifica o concuerda a la categorización con la escala MRC, arrojando promedios de 1,90 y 1,83 para los sexos femenino y masculino respectivamente, donde la disnea producto del gran esfuerzo físico; no obstante, un 38,09\% (8/21) de los casos presentaron severidades de categoría 3 y 4 . Otro indicador del compromiso respiratorio es las medias de distancia recorridas van disminuyendo significativamente hasta el grupo con IFB F clasificados como severa donde solo alcanzaron 374,10 y $301,14 \mathrm{~m}$ en femeninas y masculinos respectivamente, con valores porcentuales de $19,04 \%$ (4/21) para la categoría moderado y de $23,80 \%(5 / 21)$ para severo.

Tabla 4. IFB B e IFB F con prueba de la caminata en pacientes COVID-19 con hipertensión pulmonar

\begin{tabular}{llccccccccc}
\hline & & \multicolumn{3}{c}{ Femenino N=23 } & \multicolumn{4}{c}{ Masculino N=6 } \\
\cline { 3 - 9 } & & Media & Des Est & Max & Min & Media & Des Est & Max & Min \\
\hline \multirow{2}{*}{ Escala Esfuerzo de Borg } & Reposo (IFB B) & 1,14 & 1,64 & 5 & 0 & 1,95 & 2,15 & 5 & 0 \\
\multirow{2}{*}{ Escala Disnea MRC } & PCT6 (IFB F) & 3,38 & 2,74 & 8 & 0 & 3,53 & 3,32 & 8 & 0 \\
Distancia & & 1,90 & 1,43 & 4 & 0 & 1,83 & 1,66 & 4 & 0 \\
& Recorrida & 374,10 & 94,45 & 574 & 162 & 301,14 & 217,45 & 574 & 94 \\
& Esperada & 441,93 & 81,14 & 606 & 266 & 348,77 & 226,07 & 606 & 81
\end{tabular}

PCT6: Prueba de la caminata en 6 minutos.

IFB B: Índice de fatiga de Borg antes del ejercicio o basal.

IFB F: Índice de fatiga de Borg después del ejercicio o final.

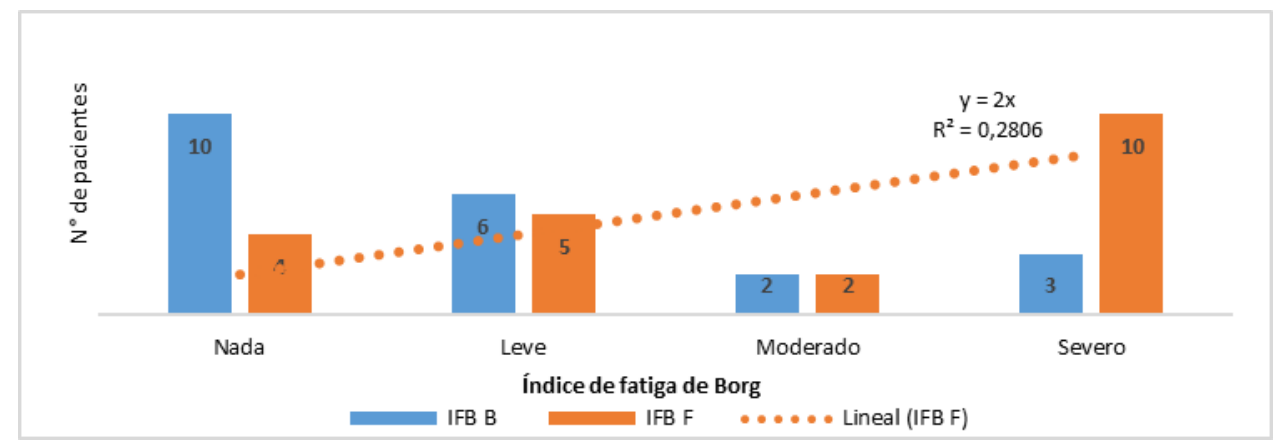

Figura 2. Categorización IFB B e IFB F con prueba de la caminata en pacientes COVID-19 con hipertensión pulmonar

En la tabla 5 se expone la relación entre el IFB F y la SpO2., $65.52 \%$ (19/29) de los pacientes se mantuvo con SpO2 conservada (disminuye menos de un $4 \%$.) después de la PCT6, de ellos el 48,24 \% presentó sensación de fatiga de moderada a severa. Al contrastar en los pacientes que desaturaron el 50,00\% (5/5) tuvieron fatiga severa. 
\% no presentó sensación de fatiga después del ejercicio, solo el 11,76 \% tuvo un IFB F severo.

Tabla 5. Relación entre IFB F y saturación de oxígeno en pacientes COVID-19 con hipertensión pulmonar

\begin{tabular}{lcccc}
\hline \multirow{2}{*}{ IFB F } & \multicolumn{2}{c}{ Saturación conservada } & \multicolumn{2}{c}{ Desaturación } \\
\cline { 2 - 5 } & $\mathbf{N}$ & $\mathbf{\%}$ & $\mathbf{N}$ & $\mathbf{\%}$ \\
\hline Nada & 2 & 6,90 & 2 & 6,90 \\
Leve & 3 & 10,34 & 3 & 10,34 \\
Moderada & 6 & 20,69 & & \\
Severa & 1 & 3,45 & 4 & 13,79 \\
Sin clasificar & 7 & $\mathbf{2 4 , 1 4}$ & 1 & 3,45 \\
\hline Total & $\mathbf{1 9}$ & $\mathbf{6 5 , 5 2}$ & $\mathbf{1 0}$ & $\mathbf{3 4 , 4 8}$ \\
\hline
\end{tabular}

\section{Discusión}

Los pacientes HAP son de peor pronostico en comparación a otras patologías, en el caso de COVID persistente con esta comorbilidad, repercuten un desafio para mantener la calidad de vida diaria y tras el ejercio, como lo cita Ryan et al., (2020), pudiéndose deber a la patología que se desencadena posterior a la vaspconstricción de la arteria pulmonar que conduce a la reducción de oxigeno en la sangre como se demostró en este estudio en un 34,48\% de los casos, como lo sugiere Avellanas Chavala, (2020); otro aspecto a resaltar son los daños endoteliales con las posibles consecuencias tromboticas.

Aunque dentro de los factores de riesgo de la HAP, no se reconocer al sexo, como lo señala D'Alonzo et al., (2012) en este estudio el sexo femenino represento en este estudio el 79,31\%, y un amplio rango de edad desde la adolescencia a la ancianidad (70 años) discrepando con estos mismos autores, clásicamente, la HPI era diagnosticada en torno a los 30 años de edad, pero Humbert et al., (2006); Badesch et al.,(2010) y Escribano-Subias et al., (2012) señalan la tendencia a diagnosticar la enfermedad más tardíamente, con una edad media sobre los 50 años.

Aunque aun en estudio, el sobrepeso es u factor que incrementa el riesgo en COVID-19, PORQUE puede aumentar las dificultades respiratorias. Otra teoría es que la obesidad puede aumentar las posibilidades de que una persona experimente la "tormenta de citoquinas", una complicación del sistema inmunológico que puede representar una amenaza para la vida de un paciente con COVID-19. Por último, tener más grasa puede ayudar al virus a permanecer más tiempo, debido a las propiedades moleculares de las células grasas y de este virus en particular", explica el Simonnet et al., (2021); que los pacientes franceses con obesidad severa (IMC $\geq 35$ ) requieren con más frecuencia de ventilación mecánica invasiva, frente a los pacientes delgados, independientemente de la edad, el sexo, la diabetes y la hipertensión arteria. En el mismo sentido, un estudio de 4.103 pacientes con COVID-19 en Nueva York asocia significativamente la obesidad con la necesidad de hospitalización y el estado crítico de los pacientes (cuidados intensivos, ventilación mecánica y/o muerte), independientemente de otras comorbilidades.

La dificultad respiratoria expresada en el IFB B categorizan entre 0 y 2 (nulos a muy suaves) y $\mathrm{R}^{2}$ alto $(0,7993)$ explica la percepción de malestar respiratorio que aparece con niveles de actividad que normalmente no causan este tipo de malestar (actividad diaria), y puede ponderarse por el nivel de actividad necesario para que se desencadene, similarmente lo reportan Avellanas Chavala, (2020); Arnanz González et al., (2021) y Sanz Almazán et al., (2021). Al aplicar la Escala de Borg modificada nos permitio valorar y cuantificar de manera sencilla y reproducible una disnea en reposo o tras la actividad diaria. Para ello se puede utilizar la escala de 10 puntos que permite calificar sensaciones respiratorias específicas (por ejemplo, dificultad inspiratoria, esfuerzo de la respiración, dificultad espiratoria, falta de aire, etc.) o más generales (por ejemplo, dificultad respiratoria, falta de aliento). Finalmente, IFB nos permitio comparar de manera fiable la intensidad de la disnea de esfuerzo con un mismo nivel de actividad física (ritmo de actividad, saturación de oxigeno) que, tras la PCT6 con sensación de fatiga severa tras ejercicio, y el $\mathrm{R}^{2}$ nos revela la relación de intensidad de esfuerzo versus demanda respiratoria traspolando a los pacientes a categoría 3 o 4, de acuerdo con Arnanz González et al., (2021) y Sanz Almazán et al., (2021). Esta escala fiable y reproducible en sujetos sanos y en pacientes con enfermedades respiratorias crónicas que puede utilizarse en COVID persistente. y sugiere su asociación de las intervenciones farmacológicas capaces de reducir la magnitud (y la duración) del esfuerzo respiratorio con la reducción de la intensidad de la disnea en COVID persistente.

Escala Disnea MRC en este estudio nos brindo la utilidad de correlacionar la PCT6 que expreso que a mayor intensidad de la actividad física es directamente proporciobal a la magnitud de la disnea, de amplio uso en el ámbito clínico en el que está validada, permitio ajustar los síntomas contados por el paciente a uno de estos estadios en diferentes esrtadios de acuerdo a la dificultad respiratoria. Por otra parte, la opinión de los pacientes se considera clave a la hora de elegir las escalas que más se ajusten a sus necesidades. Aunque las escalas y cuestionarios planteados miden bloques sintomáticos diferentes, se pueden extraer conclusiones transversales y comunes que pueden aplicarse a la hora de crear una escala integral, las fluctuaciones sintomáticas que pueden observare, incluso, en el mismo día, la situación 
clínica previa a COVID-19, y la consideración conjunta de todos los síntomas de forma específica y desde diferentes puntos de vista y situaciones. Cualquier escala útil en este contexto deberá ser sencilla y fácil de usar.

\section{Conflictos de intereses}

Ninguno para declarar

\section{Agradecimiento}

Ninguno

\section{Referencias}

Arnanz González, I., Martínez del Valle, M., Recio Garcíac, S., Blasco Redondod, R., Pérez de Inestrosae, T. B. \& Sanz Almazánf, M. (2021). Las escalas en la COVID-19 persistente. Med Gen Fam. 10(2), Revisión. Disponible en: http://mgyf.org/las-escalas-en-la-covid-19-persistente/ (Acceso mayo 2021)

Avellanas Chavala M. L. (2020). Pulmonary arterial hypertension and COVID-19. Hipertensión arterial pulmonar y COVID-19. Medicina intensiva, 44(9):577-579. https://doi.org/10.1016/j.medin.2020.05.005

Badesch, D.B., Raskob, G.E., Elliott, C.G., Krichman, A.M., Farber, H.W., Frost, A.E., Barst, R.J., Benza, R.L., Liou, T.G., Turner, M., Giles, S., Feldkircher, K. , Miller, D.P. \& McGoon, M.D. (2010). Hipertensión arterial pulmonar: características basales del Registro REVEAL. Chest , 137 (2):376-387. https://doi.org/10.1378/chest.09-1140

Carfì, A., Bernabei, R., Landi, F., \& Gemelli, A. (2020). Persistent symptoms in patients after acute COVID-19. JAMA, 324(6):603-605. https://doi.org/10.1001/jama.2020.12603

Castro Garcia, R.G., Sánchez Acuña, E.J., Moncayo Valencia, C.J., Anzules Cuzco, L. N., Rubira Clavijo, A.J., Burgos Jurado. M. C., Pazmiño Chancay, M. J., Carreño Ramos, J. E. \& Morocho Anchatuña, J.R. (2021). Intubación rápida en pacientes críticos COVID-19 en Guayaquil Ecuador, 2020. Boletín de Malariología y Salud Ambiental, 61(2): 292-298. https://doi.org/10.52808/bmsa.7e5.612.019

D'Alonzo, G. E., Barst, R. J., Ayres, S. M., Bergofsky, E. H., Brundage, B. H., Detre, K. M., Fishman, A. P., Goldring, R. M., Groves, B. M., \& Kernis, J. T. (1991). Survival in patients with primary pulmonary hypertension. Results from a national prospective registry. Annals of internal medicine, 115(5): 343-349. https://doi.org/10.7326/0003-4819-115-5-343

Díaz E., Amézaga Menéndez R., Vidal Cortés P., Escapa M.G., Suberviola B., Serrano Lázarof A., Marcos Neirag P., Quintana Díaz M. \& Catalán González M. (2021). Tratamiento farmacológico de la COVID-19: revisión narrativa de los Grupos de Trabajo de Enfermedades Infecciosas y Sepsis (GTEIS) y del Grupo de Trabajo deTransfusiones Hemoderivados (GTTH). Medicina Intensiva. 45(2,): 104-121. https://doi.org/10.1016/j.medin.2020.06.017

Dyspnea. Mechanisms, assessment, and management: a consensus statement. American Thoracic Society. (1999). American journal of respiratory and critical care medicine, 159(1):321-340. https://doi.org/10.1164/ajrccm.159.1.ats898

Escribano-Subias, P., Blanco, I., López-Meseguer, M., Lopez-Guarch, C. J., Roman, A., Morales, P., Castillo-Palma, M. J., Segovia, J., Gómez-Sanchez, M. A., Barberà, J. A., \& REHAP investigators (2012). Survival in pulmonary hypertension in Spain: insights from the Spanish registry. The European respiratory journal, 40(3):596-603. https://doi.org/10.1183/09031936.00101211

Gladwin M. T. \& Levine, A. R. (2020). Hipertensión pulmonary. Manual MSD Versión para profesionales. Disponible en: $\quad$ https://www.msdmanuals.com/es-ve/professional/trastornos-pulmonares/hipertensi\%C3\%B3npulmonar/hipertensi\%C3\%B3n-pulmonar (Acceso enero 2021)

Humbert, M., Sitbon, O., Chaouat, A., Bertocchi, M., Habib, G., Gressin, V., Yaici, A., Weitzenblum, E., Cordier, J. F., Chabot, F., Dromer, C., Pison, C., Reynaud-Gaubert, M., Haloun, A., Laurent, M., Hachulla, E., \& Simonneau, G. (2006). Pulmonary arterial hypertension in France: results from a national registry. American journal of respiratory and critical care medicine, 173(9):1023-1030. https://doi.org/10.1164/rccm.200510-1668OC

Martín Lesende, I., Álvarez Solarm Mm, Benítez del Rosario, M.A., Espinosa Almendro, J.M., Gorroñogoitia Iturbe, A. \&, Muñoz Cobos F, (2016). Atención a las personas mayores desde la Atención Primaria. Grupo de Trabajo de Atención al Mayor de la semFYC. Sociedad Española de Medicina de Familia y Comunitaria. 389 P. 
Disponible en: https://www.semfyc.es/wp-content/uploads/2016/05/Atencion-a-las-personas-mayores.pdf (Acceso enero 2021)

Mularski, R. A., Reinke, L. F., Carrieri-Kohlman, V., Fischer, M. D., Campbell, M. L., Rocker, G., Schneidman, A., Jacobs, S. S., Arnold, R., Benditt, J. O., Booth, S., Byock, I., Chan, G. K., Curtis, J. R., Donesky, D., HansenFlaschen, J., Heffner, J., Klein, R., Limberg, T. M., Manning, H. L., ... ATS Ad Hoc Committee on Palliative Management of Dyspnea Crisis (2013). An official American Thoracic Society workshop report: assessment and palliative management of dyspnea crisis. Annals of the American Thoracic Society, 10(5):S98-S106. https://doi.org/10.1513/AnnalsATS.201306-169ST

Rose V. L. (1999). American Thoracic Society issues consensus statement on dyspnea. American family physician, 59(11), 3259-3260.

Ryan, J.J., Melendres-Groves, L., Zamanian, R.T., Oudiz, R.J., Chakinala, M.., Rosenzweig, E.B. \& GombergMaitland, M. (2020). Atención de pacientes con hipertensión arterial pulmonar durante la pandemia de $\begin{array}{lllll}\text { coronavirus } & \text { (COVID-19). } & \text { Circulación } & \text { pulmonar, } 2045894020920153 .\end{array}$ https://doi.org/10.1177/2045894020920153

Sanz Almazán, M. (2021). COVID-19 persistente, utilidad y características de las escalas de valoración clínica según las percepciones de los enfermos. Novedades SIIC Salud. Disponible en: https://www.siicsalud.com/dato/resiiccompleto.php/167215 (Acceso mayo 2021)

Sanz Almazán. M., Pérez de Inestrosa, T.B., Blasco Redondo, R., Martínez del Valle, M., Recio García, S. \& Arnanz González, I. (2021). Experiencia del paciente afectado por COVID-19 persistente acerca de la utilidad y características de las escalas de valoración clínica de los síntomas derivados de su enfermedad. Med Gen Fam, 10(2): 101-103. http://dx.doi.org/10.24038/mgyf.2021.025

Simonellia, C., Paneronia, M., Vitaccaa, M. \& Ambrosino, N. (2021). Measures of physical performance in COVID-19 patients: a mapping review. Pulmonology 000 (xxxx):1-11. https://doi.org/10.1016/j.pulmoe.2021.06.005

Sociedad Española de Médicos Generales y de Familia (SEMG). (2000). COVID-19 persistente. Disponible en: https://www.semg.es/images/2020/Noticias/20201111_Resultados_Encuesta_COVID_Persistente.pdf (Acceso enero 2021)

Simonnet A., Chetboun M., Poissy J., Reverdy V., Noulette J., Duhamel A. (2020). High prevalence of obesity in severe acute respiratory syndrome coronavirus-2 (SARS-CoV-2) requiring invasive mechanical ventilation. Obesity. https://doi.org/10.1002/oby.22831 